\title{
Research Paper: The Effects of Continuous Cognitive Task on Postural Control of People With Anterior Cruciate Ligament Reconstruction Using Balance Error Scoring System
}

\section{Payam Ahmadi $^{1}$ (ㅇ, *Reza Salehi ${ }^{2}$ (), Mohammad Mehravar ${ }^{3}$, Shahin Goharpey ${ }^{4}$}

1. PhD. Student of Physiotherapy, Musculoskeletal Rehabilitation Research Center, Ahvaz Jundishapur University of Medical Sciences, Ahvaz, Iran. 2. Associate Professor of Physiotherapy, Department of Rehabilitation Management, School of Rehabilitation Sciences, Rehabilitation Research Center, Iran University of Medical Sciences, Tehran, Iran.

3. Master of Science in Mechanical Engineering, Musculoskeletal Rehabilitation Research Center, Ahvaz Jundishapur University of Medical Sciences, Ahvaz, Iran. 4. Assistant Professor of Physiotherapy, Musculoskeletal Rehabilitation Research Center, Ahvaz Jundishapur University of Medical Sciences, Ahvaz, Iran.

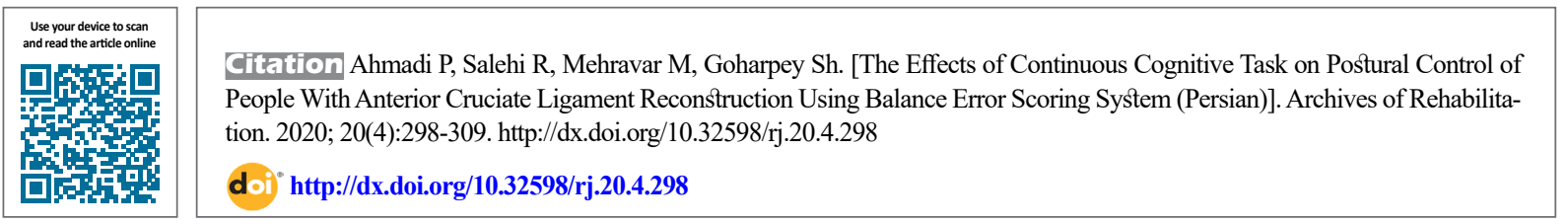

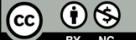

Received: 10 Apr 2019 Accepted: 23 Sep 2019 Available Online: 01 Jan 2020

Keywords:

Anterior cruciate ligament reconstruction, Postural balance, Balance error scoring system, Attention

\section{A B STRACT}

Objective People with Anterior Cruciate Ligament $(A C L)$ reconstruction need to have almost automatic postural control to focus on their athletic skills. Continuous cognitive task is a method for removing attention from postural control. The effects of continuous cognitive task on postural control of people with ACL reconstruction has been assessed by laboratory tools; however, its effect using balance error scoring system is still unknown. Therefore, the present study aimed to investigate the effects of continuous cognitive task on the postural control of individuals with ACL reconstruction using the balance error scoring system.

Materials \& Methods The present study is a case-control study using simple non-probability sampling. Participants included 20 male football players with ACL reconstruction (13.90 \pm 4.15 months after surgery) and 20 healthy men. They were matched for age, sex, height, weight, body mass index, dominant leg, education and activity levels. Tegner activity level scale was used to determine activity level of participants. Postural control was evaluated under single-leg stance test (with treated leg) on firm and foam surfaces with and without continuous cognitive task. Balance error scoring system was used to assess the balance control. In the condition without cognitive task, no feedback was provided. Continuous cognitive task consisted of 10 three-digit numbers each presented with a 2-second interval by audio tape and headphone. In the cognitive condition, individuals were asked to focus on the preselected number and provide the sum of its repetitions in the end of the test. The dependent variable was the number of balance error in each condition. Balance errors included lifting hands from hip, opening eyes, stepping or swinging, lifting forefoot or heal, moving hip into more than 30 degrees of hip abduction or flexion, and remaining out of position for more than 5 seconds. We used SPSS V. 20 software for statistical analysis of data. Kolmogorov- Simonov test was performed to test the normality of data distribution. Intraclass Correlation Coefficient (ICC) was calculated to assess the relative reliability of the rater for assessing the balance error. The absolute reliability of the rater was measured using Standard Error of Measurement (SEM), Minimal Metrically Detectable Change (MMDC) and Coefficient of Variation (CV). Mixed-model of ANOVA ( 2 groups $\times 4$ stance conditions) was used to investigate the main effect and interaction effect of these factors. We used repeated measure ANOVA to determine the main effect of test condition in every each, and independent-sample t-test and effect size was used to determine the difference between groups.

\section{* Corresponding Author:}

Reza Salehi, PhD.

Address: Department of Rehabilitation Management, School of Rehabilitation Sciences, Rehabilitation Research Center, Iran University of Medical Sciences, Tehran, Iran Tel: +98 (916) 3015563

E-Mail: salehi.re@iums.ac.ir 
Results Distribution of data in all four test conditions was normal $(P>0.05)$. The rater reliability was excellent for calculating the balance error in all test conditions (ICC $>0.90, \mathrm{MMDC}<\% 10$ and $\mathrm{CV}<\% 5$ ). Interaction between group and test condition was significant $(\mathrm{F}=11.59, \mathrm{P}<0.01)$. Result of independent-sample t-test showed that subjects had a higher balance error than healthy subjects under single-leg stance test on foam without cognitive task $(\mathrm{F}=2.01, \mathrm{P}<0.01)$. In this condition, the effect size also showed that difference between groups was large $(>0.8)$. The main effect of test condition in subjects showed that the continuous cognitive task reduced balance error while standing on both firm and foam surfaces $(P<0.01)$, while in healthy subjects, the cognitive task had no effect on the balance $(P>0.05)$.

Conclusion It can be concluded that male football players with $\mathrm{ACL}$ reconstruction had poorer stability compared to healthy subjects in performing single-leg stance test on foam surface without continuous cognitive task. Moreover, continuous cognitive task improved balance control in ACL subjects, while it had no effect on the balance of healthy subjects. 
This Page Intentionally Left Blank 


\title{
ارزيابى تأثير تكليف شناختى يبوسته بر كنترل ياسجر افراد با بازسازى ليخكامان مثقاطع قدامى با استفاده از سيستم امتيازدهى خطائ تعادل
}

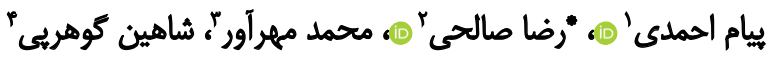

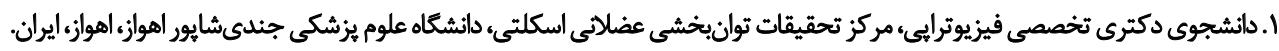

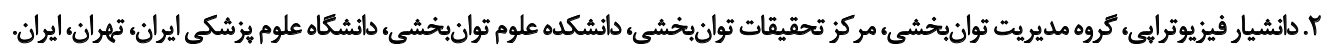

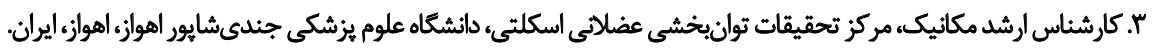

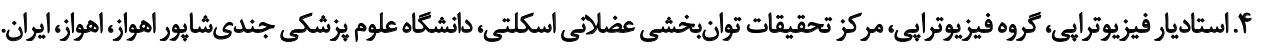

\begin{abstract}
حكيد

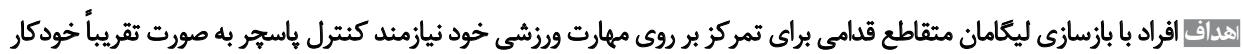

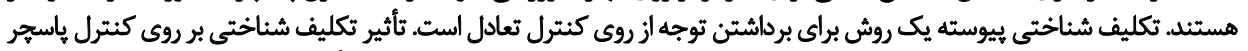

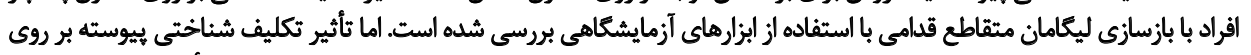

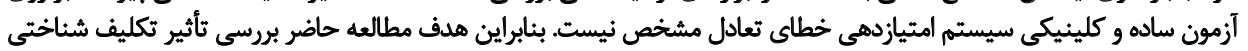

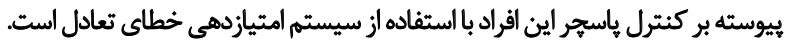

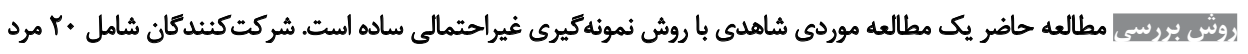

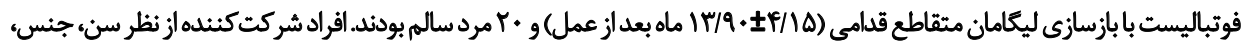

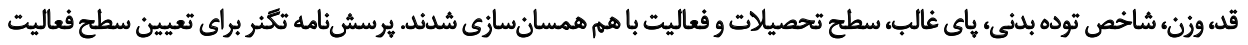

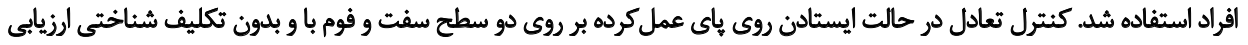

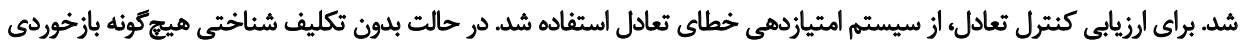

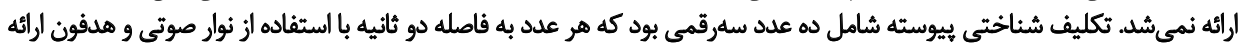

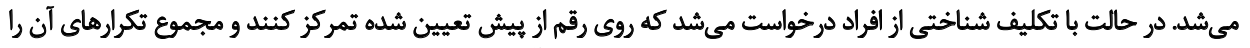

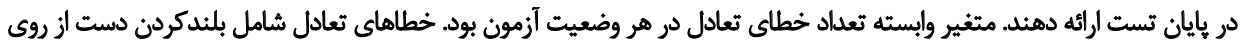

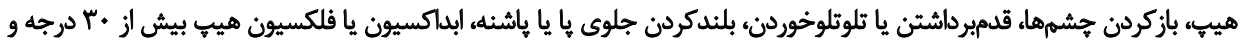

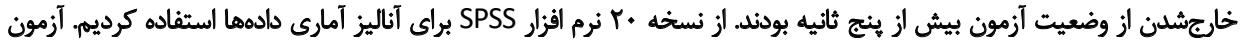

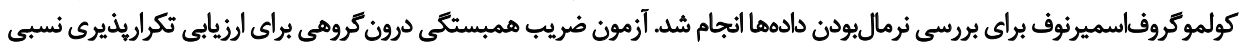

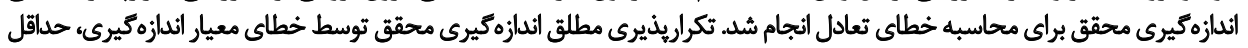

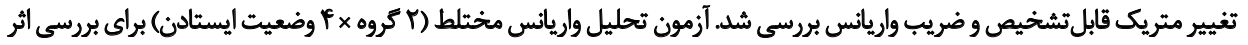

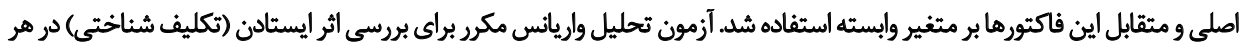

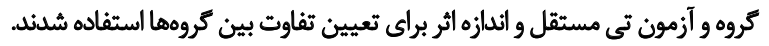

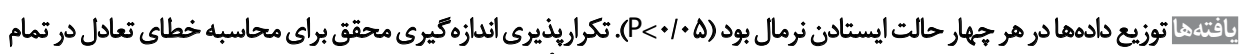

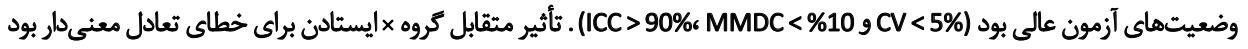

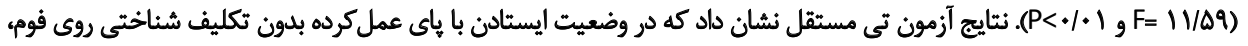

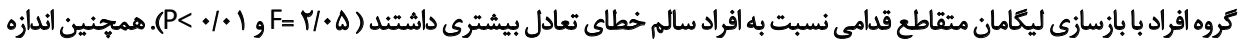

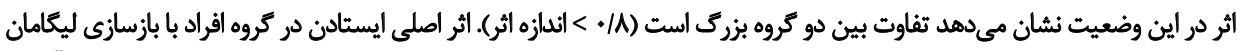

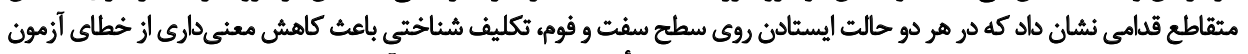

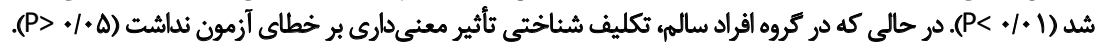

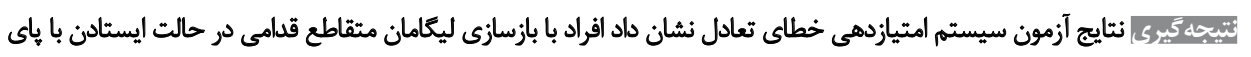

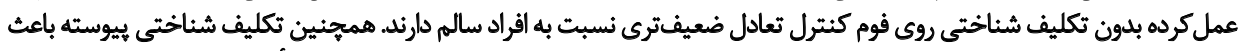

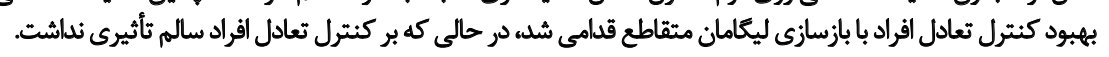

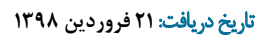

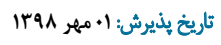


خطاى تعادل است. فرضيات ما اين است كه تكليف شناختي مقدمه

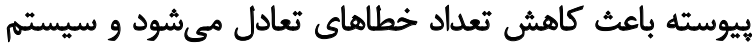

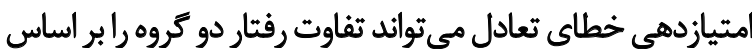
توجه نشان دهد. ووشُ بروسي

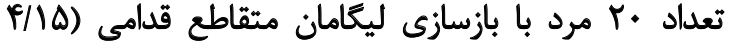

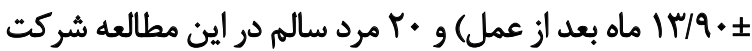

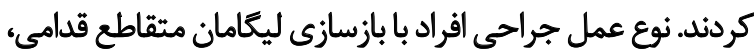

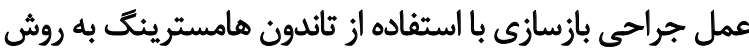

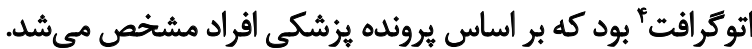

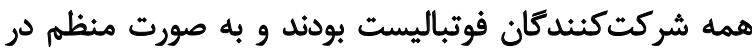
فعاليتهاى ورزشى شركت داشتيند.

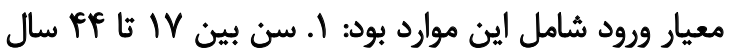

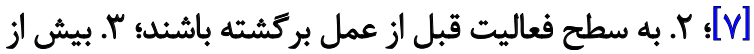

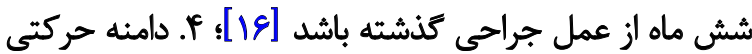

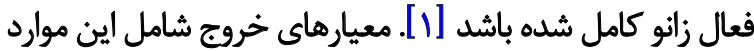

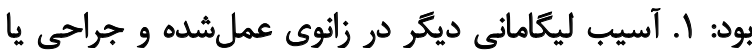

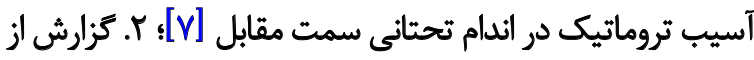

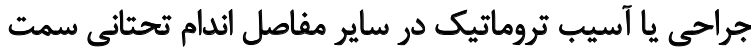

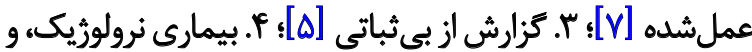

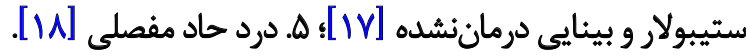
كروه افراد سالم هيج تونه آسيب يا بيمارى عضلاتى اسكلتى

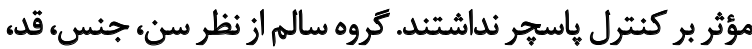

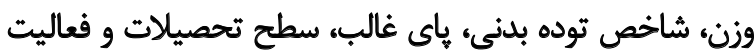

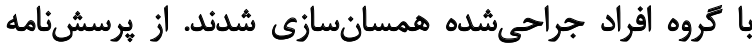

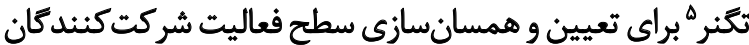

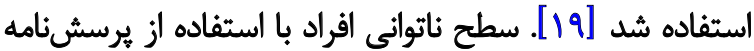

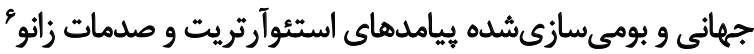

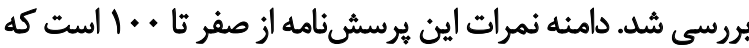

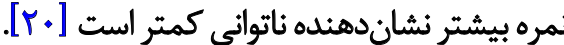
از روش سيستم امتيازدهى خطاى تعادل براى بررسى كنترل

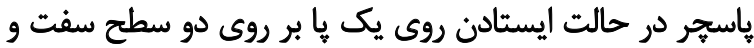

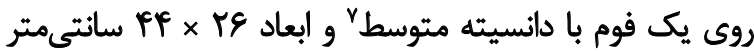

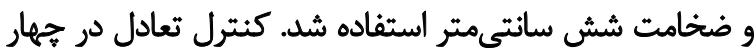

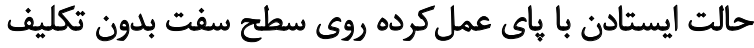

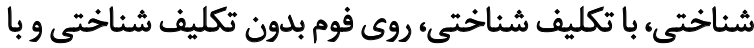

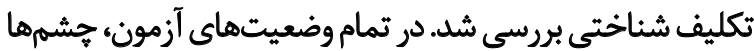

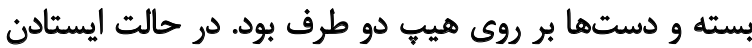

\section{Hamstring tendon autograft}

5. Tegner

6. Knee injury osteoarthritis outcome score

7. Mambo balance pad
اقراد با بازسازى ليعامان متقاطع قدامى' براى انجام فعاليت

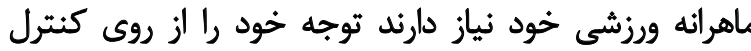

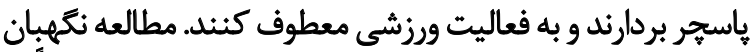

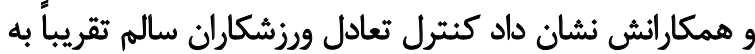
صورت خودكار است [1]]. در مطالعات اخير براي برداشتين تورجيه

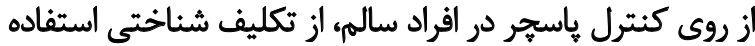

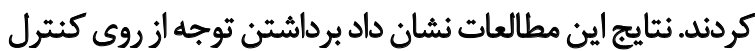

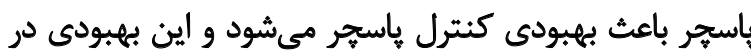

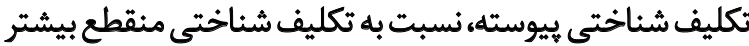

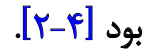

تكليف شناختى ويوسته نياز مداوم به توجه دارد و مى تواند

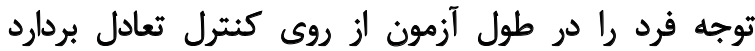

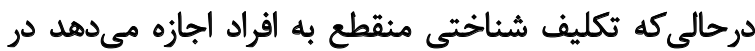

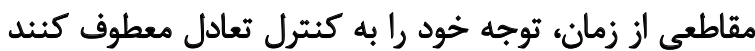

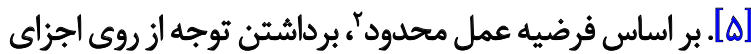

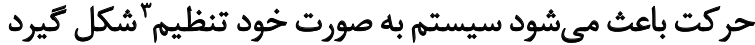

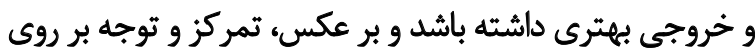

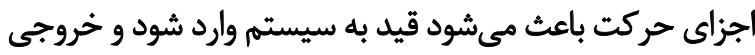

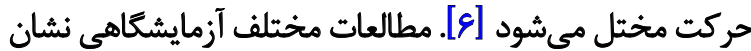

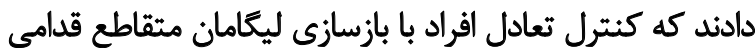

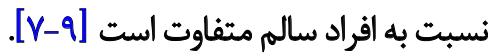

همجنين مطالعاتى كه تأثير تكليف شناختى رابر كنترل تعادل

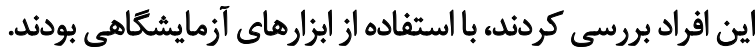

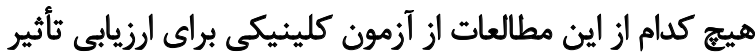

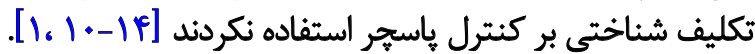

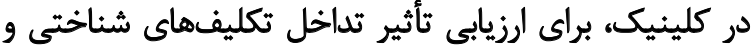

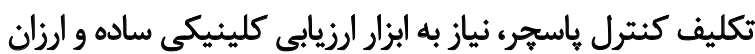

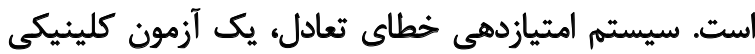

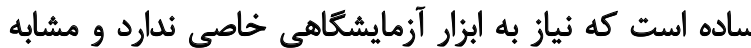

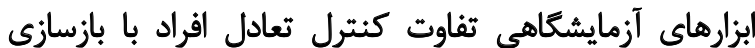

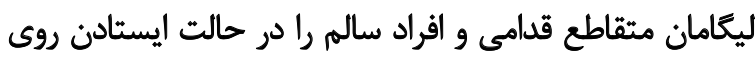

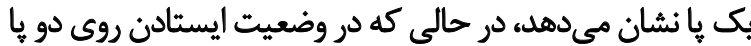

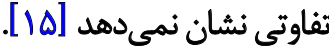

بنابراين هدف اول در مطالعه حاضر بررسى ثأثير تكليف

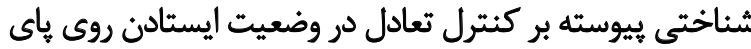

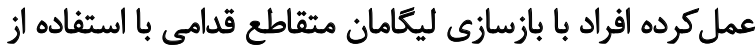

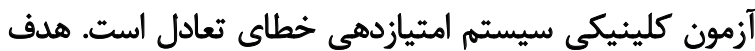

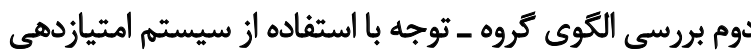


تغيير متريك قابلتشخيص" و ضريب واريانس ها بررسى شد.

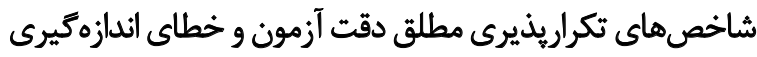

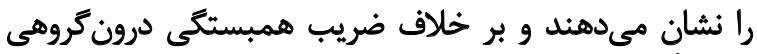

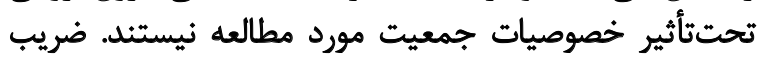

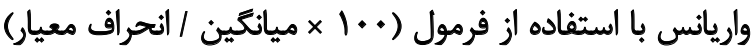

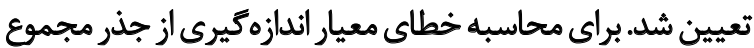

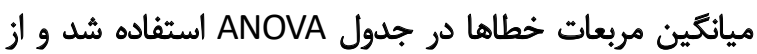

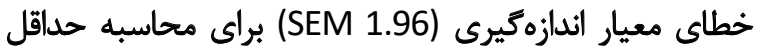

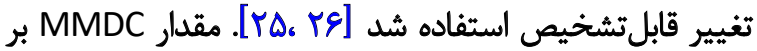

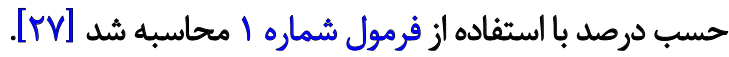

\section{$100 \times$ mean/ MMDC=MMDC\% 100}

آزمون تحليل واريانس مختلط" (ب كروه × f سطح ايستادن)

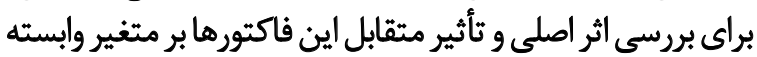

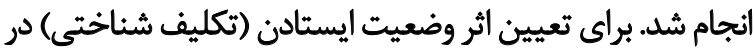

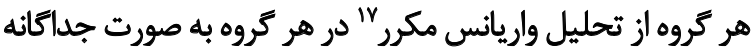

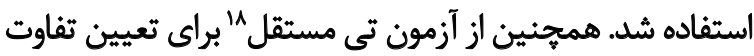
بين گروهها در هر وضعيت ايستادن استفاده شد.

يافتهاهنا

جدول شماره ا اطلاعات جمعيتشناسى، سطح فعاليت

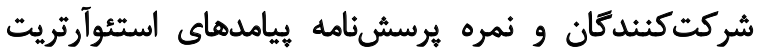

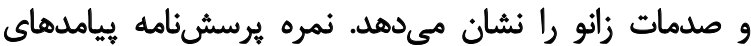

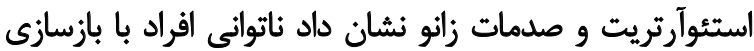

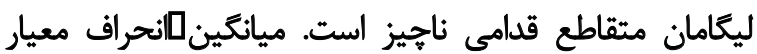

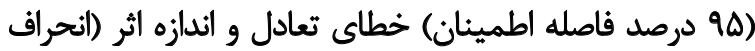

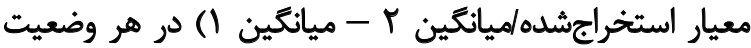

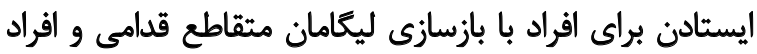

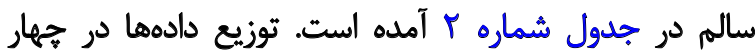

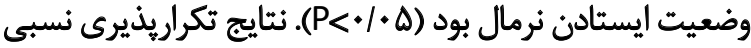

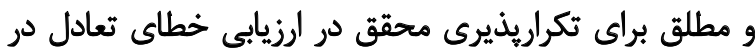

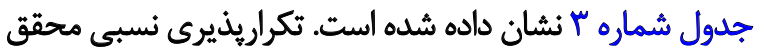

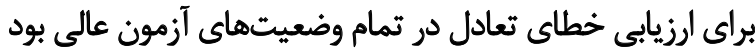
تكريذي (ICC 9•)

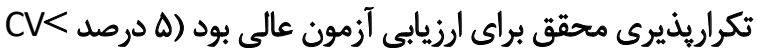

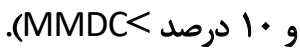

تأثير متقابل 19 تروه × ايستادن (تصويرهاى شماره | و r)

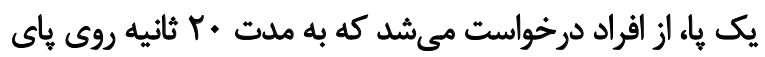

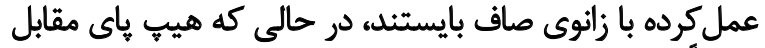

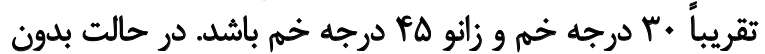

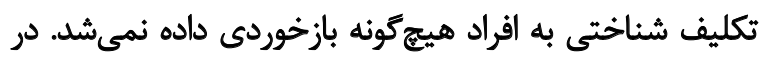

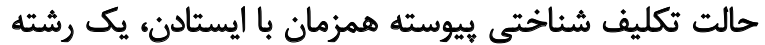

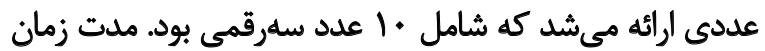

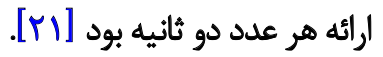

قبل از ارائه رشته عددى، يك رقم به صورت تصادفى انتخاب

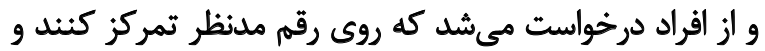

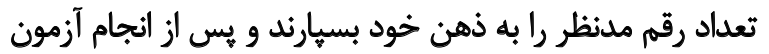

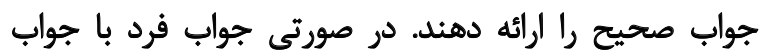

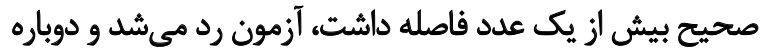

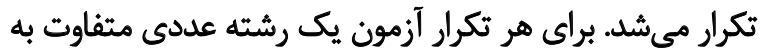

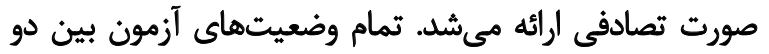

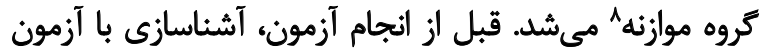

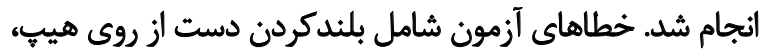

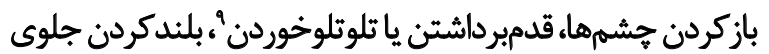

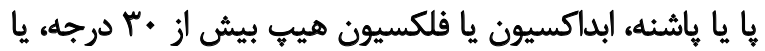

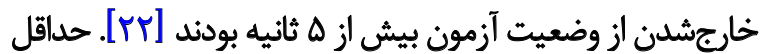

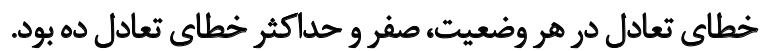

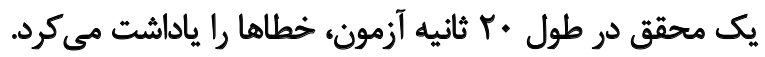

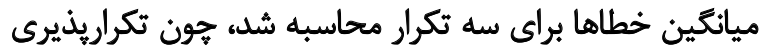

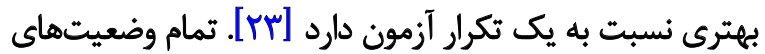

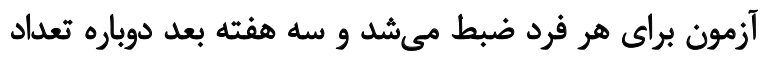

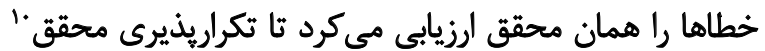

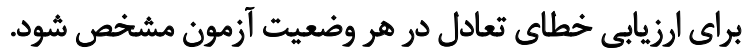

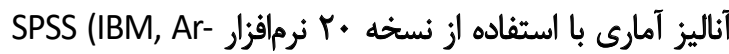
monk, NY, USA) بررسى نرمال بودن دادهها انجام شد. تكراريذيري نسبى و مطلق برئ براي

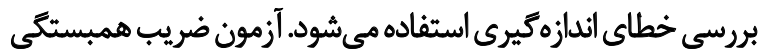

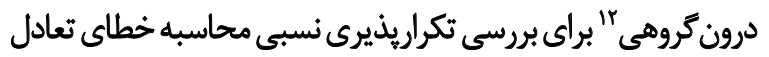

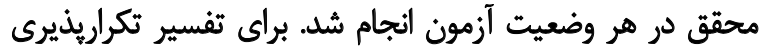

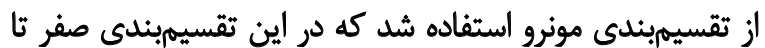

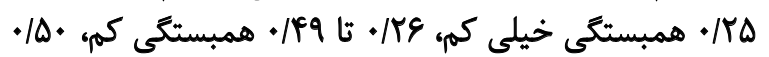

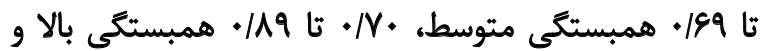

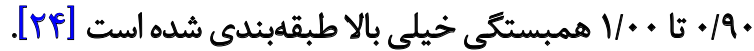

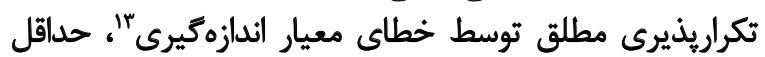

8. Counterbalance

9. Stepping or stumbling

10. Intraobserver reliability

11. Kolmogorov - smirnov

12. Intraclass Correlation Coefficient (ICC)

13. Standard Error of Measurement (SEM) 
جدول ا. ميانكين و انحراف معيار دادهاى جمعيتشئاسى، سطح فعاليت تكثنر و يرسشئامه بيامد استثوآرتريت و صدمات زانو

\begin{tabular}{|c|c|c|c|c|}
\hline $\mathbf{P}$ & أواد سالم & افراد با بازسازى ليكامان مثئاطع قدامى & \multicolumn{2}{|c|}{ متغير } \\
\hline.$/ V E$ & $r \Delta / q \cdot \pm r / Q V$ & $r E / T \cdot \pm r / Q A$ & سن (سال) & \\
\hline$\cdot M e$ & $I V N F \cdot \pm \Delta / \pi T$ & $\mid V q / \cdot \Delta \pm \varepsilon / A V$ & قد (سانتىمتر) & \\
\hline.$/ 48$ & $n / m \cdot \pm 1 \cdot / \Delta 1$ & $Y N \cdot . \pm 1 \cdot / 18$ & وزن (كيلوكرم) & دادهاى جمعيتشناسيى \\
\hline.$/ \pi$ & $r T / T \pm T / T V$ & repetrar & شاخص توده بدنى (كيلوكرم / مترمريع) & \\
\hline.$/ 44$ & $17 / 8 \cdot \pm 1 / 48$ & $\mid r / . . \pm 1 / W$ & سطح تحصيلات (سال) & \\
\hline$+/ \mu$ & $N A \cdot \pm+|8|$ & $N \& \cdot \pm+/ A r$ & سطح فعاليت تكنر & \\
\hline - & - & 1.0 & درد & \\
\hline- & - & QADET/IY & عامت & يربششنامه ييامد أستؤآرتريت و \\
\hline- & - & $1 .$. & فعاليتهاى روزمره & صلمات زأو \\
\hline- & - & $1 .$. & ورزش و تفريح & \\
\hline- & - & QWADEY/IY & كيفيت زندىى & \\
\hline
\end{tabular}

و فوم، تكليف شناختى باعث كاهش معنى دارى از خطاي آزمون

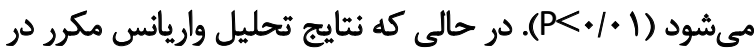

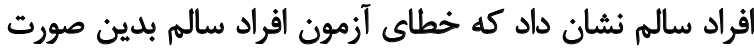

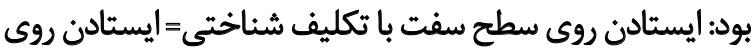

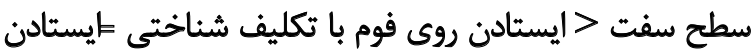

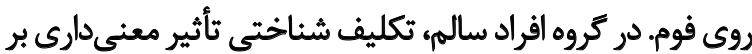

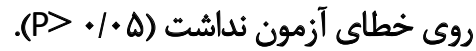

\section{بحث}

اهداف اين مطالعه: الـ بررسي تأثير تكليف شناختى بر سيستم

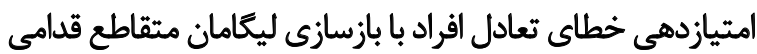

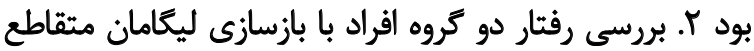

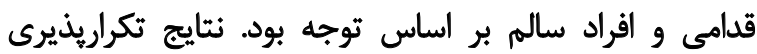

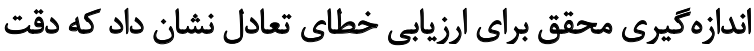

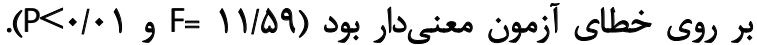

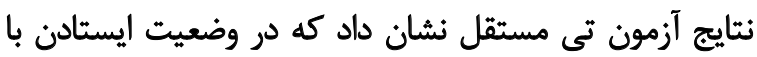

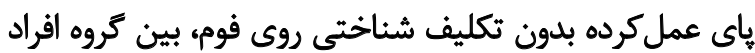

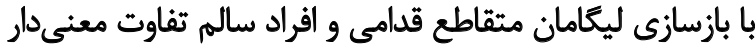

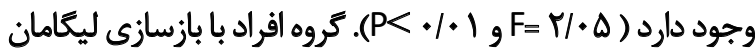

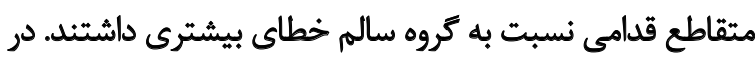

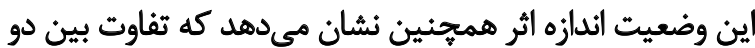
كروه بزرى است (ي| •> اندازه اثر).

نتايج تحليل واريانس مكرر نشان داد خطاى آزمون در كروه

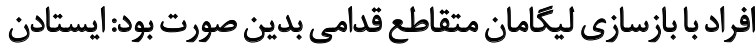

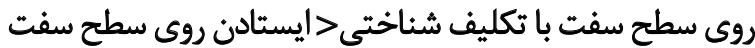

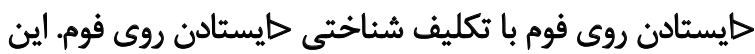
نتايج نشان مى دهد كه در هر دو حالت ايستادن روى سطح سفت

جدول r. ميانكين و انحراف معيار (ه درصد فاصله اطمينان) خطاى تعادل و اندازه اثر در هر وضعيت ايستادن

\begin{tabular}{|c|c|c|c|}
\hline \multirow{2}{*}{ اندازه اثر } & \multicolumn{2}{|c|}{ ميانكين [انحراف معيار } & \multirow{2}{*}{ وضعيت ايستادن } \\
\hline & افراد سالم & افراد با بازسازى ليكامان مثقاطع قدامى & \\
\hline$\frac{.181}{(-* / 189, \cdot / 4 \cdot)}$ & $\begin{array}{l}r / Y \wedge \pm \cdot M \\
(T / *, T / M)\end{array}$ & $\begin{array}{l}r / \Delta \cdot \pm \cdot / M \\
(T / H, T / A Y)\end{array}$ & ايستادن روى سطح سفت \\
\hline $\begin{array}{c}-\cdot / M) \\
(-\cdot / N, g / F T)\end{array}$ & $\begin{array}{l}r / T \cdot \pm \cdot / P A \\
(T / \cdot A, Y / \Delta T)\end{array}$ & $\begin{array}{l}r / T \cdot \pm \cdot / \Delta q \\
(N / A Y, Y / P V)\end{array}$ & ايستادن روى سطح سفت با تكليف شناختى \\
\hline $\begin{array}{c}1 / F r \\
(1 / . ., 1 / M)\end{array}$ & $\begin{array}{l}\Delta / Q \varepsilon_{ \pm} / \Lambda^{*} \\
\left(\Delta / \Delta \Lambda, \& / M^{\prime}\right)\end{array}$ & $\begin{array}{l}v / u \pm v / 1+ \\
(v / f e, N+q)\end{array}$ & إيستادن روى فوم \\
\hline $\begin{array}{c}.108 \\
(-.1 .8,1 / 11)\end{array}$ & $\begin{array}{c}\Delta / \& 8 \pm \cdot / q \Delta \\
(\Delta / \cdot 1, \Delta / \Delta Q)\end{array}$ & $\begin{array}{l}g / \cdot . \pm \cdot / M \\
(\Delta / \Delta M, \& / N Y)\end{array}$ & ايستادن روى فوم با تكليف شناختى \\
\hline
\end{tabular}

توانبخننى 
جدول با. نتايج مربوط به تكراريذيرى محقق در ارزيابى خطاي تعادل در وضعيتهاى مختلف ايستادن

\begin{tabular}{|c|c|c|c|c|}
\hline MMDC (\%) & SEM & $\operatorname{cv}(\%)$ & ICC & وضعيت ايستادن \\
\hline S/TE & $+/ 1 \Delta$ & $F / \Delta \Delta$ & $+/ 97(. / 98,+/ 94)$ & ايستادن روى سطح سفت \\
\hline$\Delta / v E$ & $\cdot / \pi$ & $H / . \Delta$ &.$/ Q E(. / 9 T, . / Q 4)$ & ايستادن روى سطح سفت با تكليف شناختى \\
\hline$m$. & $\cdot \pi r$ & 1/9v & $. / 41 . / 97,+/ 99)$ & ايستادن روى فوم \\
\hline r/qV &.$/ 1 \mathrm{r}$ & Ver &.$/ 41(.196, . / 99)$ & ايستادن روى فوم با تكليف شناختى \\
\hline
\end{tabular}

افراد سالم كنترل تعادل ضعيفترى داشتند [C

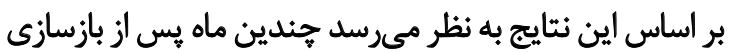

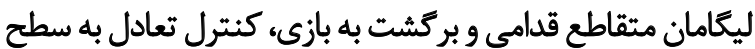

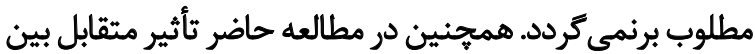

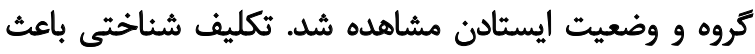

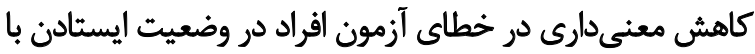

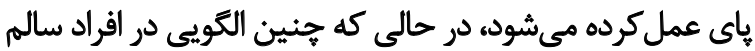

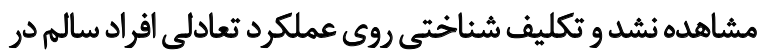

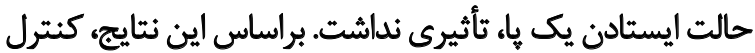

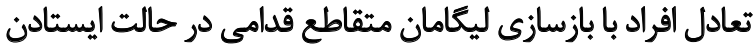

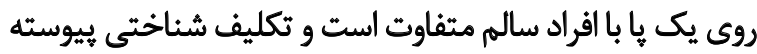

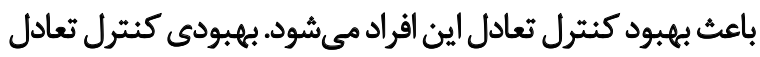

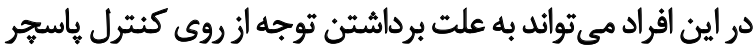

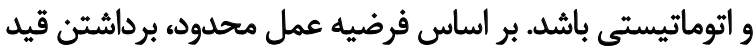

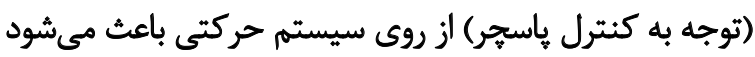

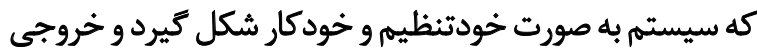

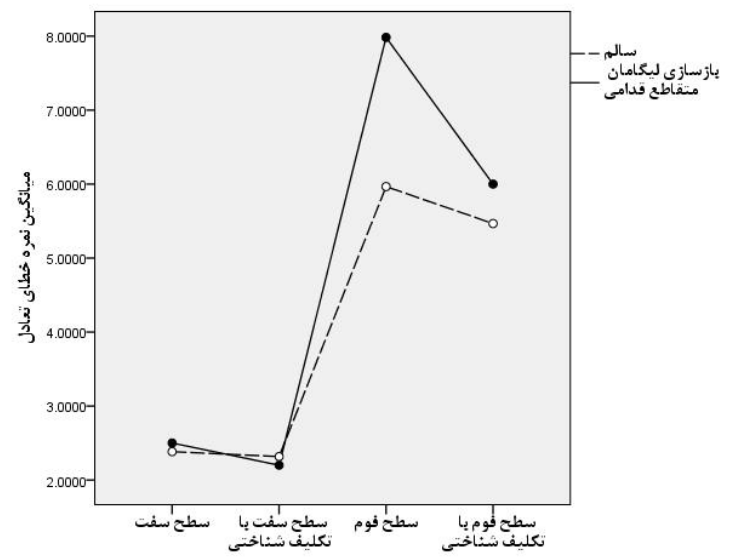

توانبخننى

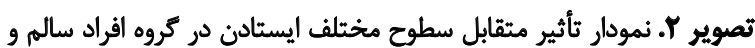

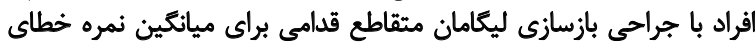

اندازهكيرى محقق براي ارزيابي خطاي تعادل مناسب است و اين

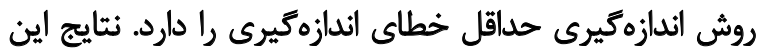

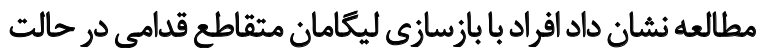

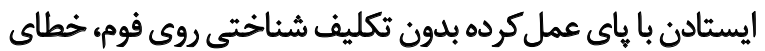

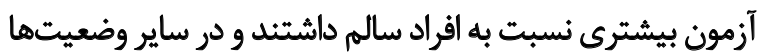
تفاوتى بين دو كروه وجود نداشت. يك مطالعه كه كنترل تعادل افراد با بازسازى ليكامان متقاطع

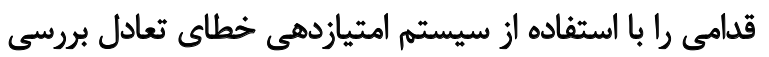

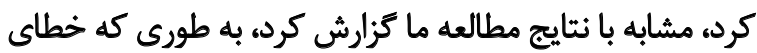

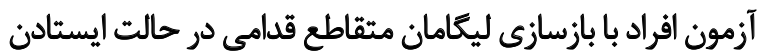

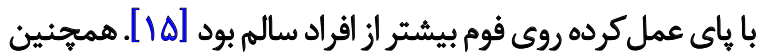

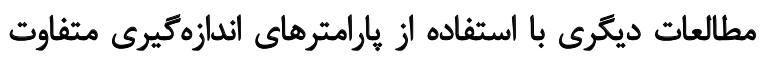

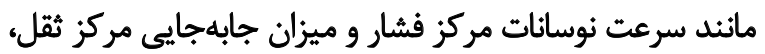

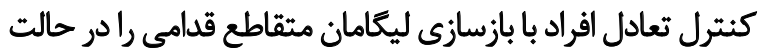

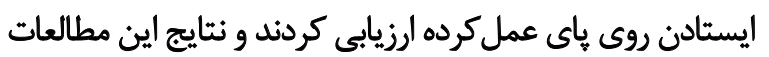

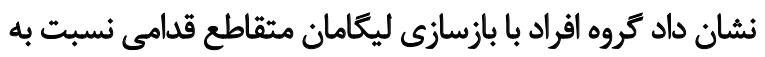

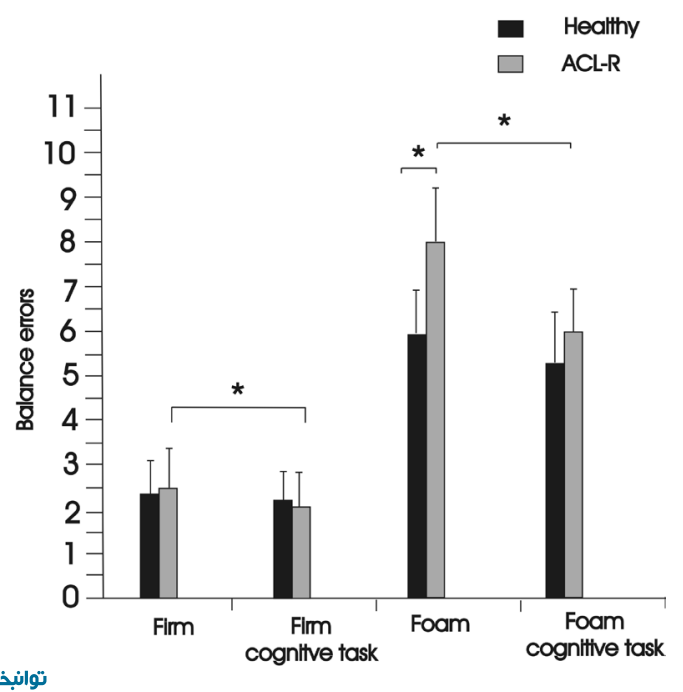

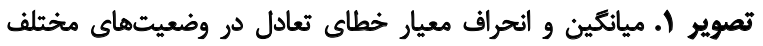

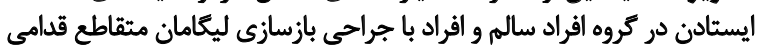

$\left({ }^{*} \mathrm{P}<\cdot 1 \cdot \Delta\right)$

سطح سفت: Firm؛ سطح سفت با تكليف شناختي: Firm cognitive task، فوم:Foam فوم با تكليف شناختى: Foam cognitive task 
ياى عملكرده بدون تكليف شناختى روى فوم، كنترل تعادل

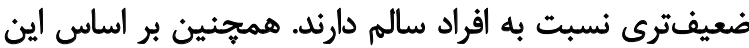

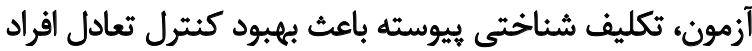

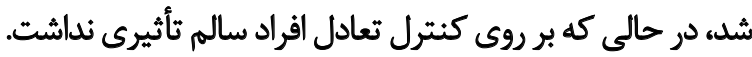

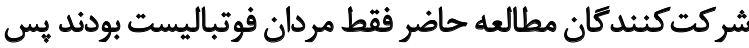

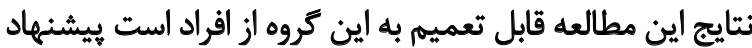

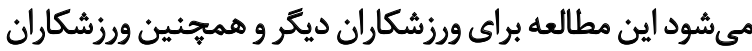

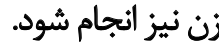

مالاحظات اخلاقي

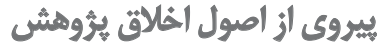

اين مطالعه توسط كميته اخلاق دانشكاه جندى إئايور اهواز

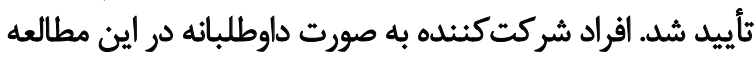

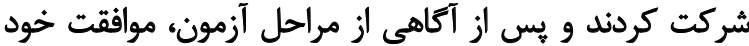

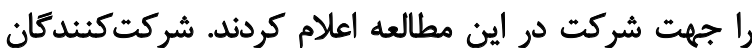

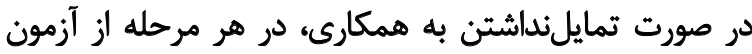

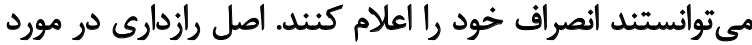
اطلاعات افراد رعايت شد.

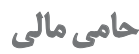

اين مقاله بخشى از رساله دكترى بيام احمدى در كروه

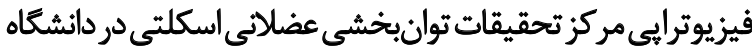

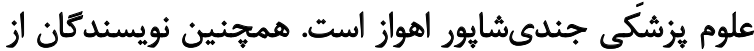

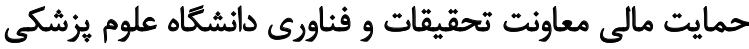
اهواز (شماره طرح 9622-PHT) برخوردار بودند.

$$
\text { مشاركت نويسندكًان }
$$

همه نويسندكان در آمادهازى مقاله سهيم بودند.

$$
\text { تشارض مثافع }
$$

نويسندكان از معاونت تحقيقات وفناورى دانشًاه علوم يزشكى اهواز بابت حمايت مالى طرح سياسكزارند.

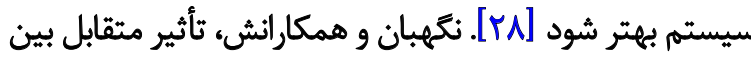

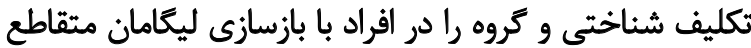

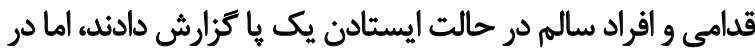

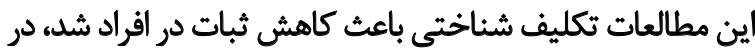

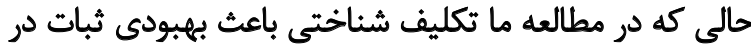
افراد با بازسازى ليعامان متقاطع قدامى شد ماليف [1].

دليل اين تفاوت ممكن است مربوط به نوع تكليف شناختى مطالعه

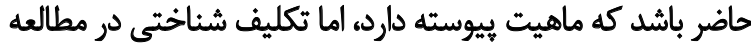
نكهببان و همكارانش ماهيت بيوسته نداشت. تكليف شئاختى منقطع

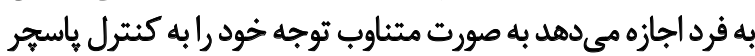

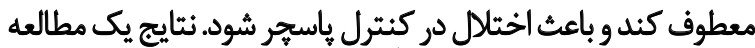

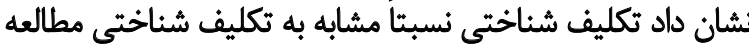

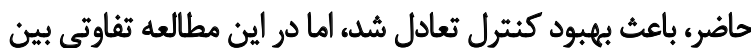

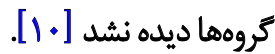

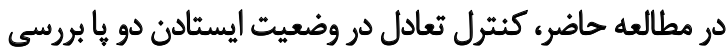

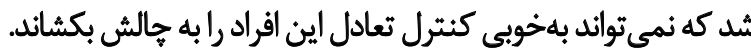

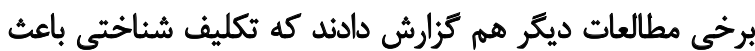

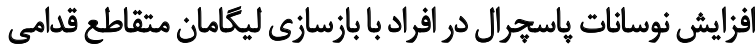

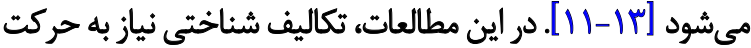

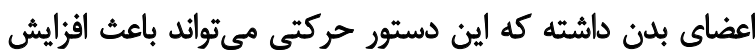

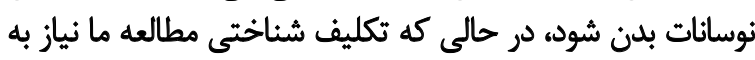

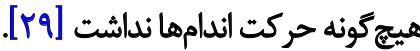
به علاوه نتايج مطالعه حاضر نشان ميدهد كه سيستم امتيازدهى

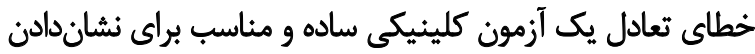

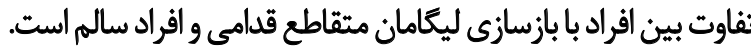

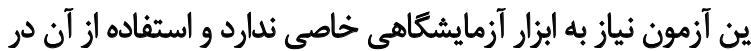

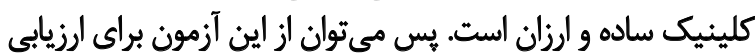

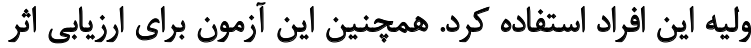

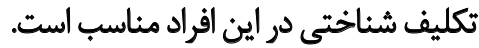

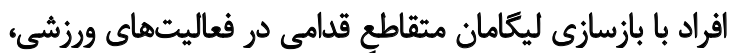

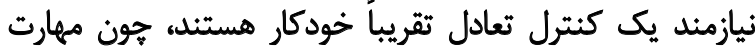

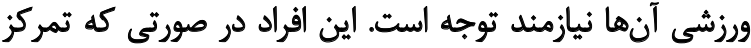

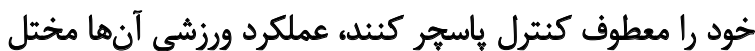

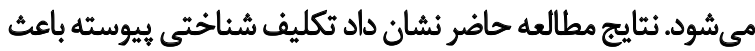

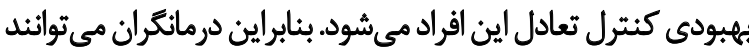

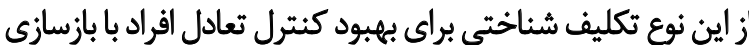

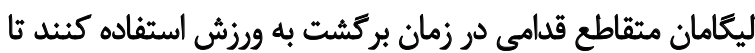

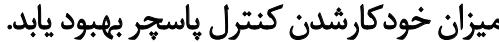
تثيجهيرى

نتايج آزمون سيستم امتيازدهى خطاي تعادل نشان داد إداد كه

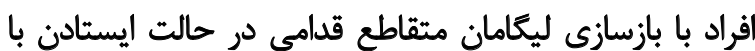




\section{References}

[1] Negahban H, Ahmadi P, Salehi R, Mehravar M, Goharpey S. Attentional demands of postural control during single leg stance in patients with anterior cruciate ligament reconstruction. Neuroscience Letters. 2013; 556:118-23. [DOI:10.1016/j.neulet.2013.10.022] [PMID]

[2] Lajoie Y, Richer N, Jehu DA, Tran Y. Continuous cognitive tasks improve postural control compared to discrete cognitive tasks. Journal of Motor Behavior. 2016; 48(3):264-9. [DOI:10.1080/0 0222895.2015.1089833] [PMID]

[3] Polskaia N, Richer N, Dionne E, Lajoie Y. Continuous cognitive task promotes greater postural stability than an internal or external focus of attention. Gait Posture. 2015; 41(2):454-8. [DOI:10.1016/j.gaitpost.2014.11.009] [PMID]

[4] Richer N, Polskaia N, Lajoie Y. Continuous cognitive task promotes greater postural stability than an internal or external focus of attention in older adults. Experimental Aging Research. 2017; 43(1):21-33. [DOI:10.1080/0361073X.2017.1258214] [PMID]

[5] Lajoie Y, Jehu DA, Richer N, Chan A. Continuous and difficult discrete cognitive tasks promote improved stability in older adults. Gait \& Posture. 2017; 55:43-8. [DOI:10.1016/j.gaitpost.2017.04.003] [PMID]

[6] Polskaia N, Lajoie Y. Reducing postural sway by concurrently performing challenging cognitive tasks. Human Movement Science. 2016; 46:177-83. [DOI:10.1016/j.humov.2015.12.013] [PMID]

[7] Zouita Ben Moussa A, Zouita S, Dziri C, Ben Salah FZ. [Single-leg assessment of postural stability and knee functional outcome two years after anterior cruciate ligament reconstruction (English, French)]. Annals of Physical and Rehabilitation Medicine. 2009; 52(6):475-84. [DOI:10.1016/j.rehab.2009.02.006] [PMID]

[8] Paterno MV, Schmitt LC, Ford KR, Rauh MJ, Hewett TE. Altered postural sway persists after anterior cruciate ligament reconstruction and return to sport. Gait \& Posture. 2013; 38(1):13640. [DOI:10.1016/j.gaitpost.2012.11.001] [PMID] [PMCID]

[9] Pahnabi GR, Akbari M, Nakhostin Ansari N, Mardani M, Ahmadi M, Rostami M. Comparison of the postural control between football players following $\mathrm{ACL}$ reconstruction and healthy subjects. Medical Journal of the Islamic Republic of Iran. 2014; 28:101. [PMID] [PMCID]

[10] Lion A, Gette P, Meyer C, Seil R, Theisen D. Effect of cognitive challenge on the postural control of patients with $\mathrm{ACL}$ reconstruction under visual and surface perturbations. Gait \& Posture. 2018; 60:251-7. [DOI:10.1016/j.gaitpost.2017.12.013] [PMID]

[11] Howells BE, Clark RA, Ardern CL, Bryant AL, Feller JA, Whitehead TS, et al. The assessment of postural control and the influence of a secondary task in people with anterior cruciate ligament reconstructed knees using a Nintendo Wii Balance Board. British Journal of Sports Medicine. 2013; 47(14):914-9. [DOI:10.1136/bjsports-2012-091525] [PMID]

[12] Mohammadirad Sh, Salavati M, Ebrahimi Takamjani I, Akhbari $B$, Sherafat Sh, Mazaheri M, et al. Intra and intersession reliabil- ity of a postural control protocol in athletes with and without anterior cruciate ligament reconstruction: A dual-task paradigm. International Journal of Sports Physical Therapy. 2012; 7(6):627-36. [PMID] [PMCID]

[13] Akhbari B, Salavati M, Ahadi J, Ferdowsi F, Sarmadi AR, Keyhani $S$, et al. Reliability of dynamic balance simultaneously with cognitive performance in patients with $\mathrm{ACL}$ deficiency and after $\mathrm{ACL}$ reconstructions and in healthy controls. Knee Surgery, Sports Traumatology, Arthroscopy. 2015; 23(11):3178-85. [DOI:10.1007/s00167-014-3116-0] [PMID]

[14] Mohammadi F, Salavati M, Akhbari B, Mazaheri M, Khorrami $\mathrm{M}$, Negahban $\mathrm{H}$. Static and dynamic postural control in competitive athletes after anterior cruciate ligament reconstruction and controls. Knee Surgery, Sports Traumatology, Arthroscopy. 2012; 20(8):1603-10. [DOI:10.1007/s00167-011-1806-4] [PMID]

[15] Smith MD, Bell DR. Negative effects on postural control after anterior cruciate ligament reconstruction as measured by the balance error scoring system. Journal of Sport Rehabilitation. 2013; 22(3):224-8. [DOI:10.1123/jsr.22.3.224] [PMID]

[16] Gokeler A, Benjaminse A, Welling W, Alferink M, Eppinga P, Otten $B$. The effects of attentional focus on jump performance and knee joint kinematics in patients after $\mathrm{ACL}$ reconstruction. Physical Therapy in Sport. 2015; 16(2):114-20. [DOI:10.1016/j. ptsp.2014.06.002] [PMID]

[17] Gianotti SM, Marshall SW, Hume PA, Bunt L. Incidence of anterior cruciate ligament injury and other knee ligament injuries: A national population-based study. Journal of Science and Medicine in Sport. 2009; 12(6):622-7. [DOI:10.1016/j. jsams.2008.07.005] [PMID]

[18] Harrison EL, Duenkel N, Dunlop R, Russell G. Evaluation of single-leg standing following anterior cruciate ligament surgery and rehabilitation. Physical Therapy. 1994; 74(3):245-52 [DOI:10.1093/ptj/74.3.245] [PMID]

[19] Negahban H, Mostafaee N, Sohani S, Mazaheri M, Goharpey $\mathrm{S}$, Salavati M, et al. Reliability and validity of the Tegner and Marx activity rating scales in Iranian patients with anterior cruciate ligament injury. Disability and Rehabilitation. 2011; 33(23-24):2305-10. [DOI:10.3109/09638288.2011.570409] [PMID]

[20] Salavati M, Mazaheri M, Negahban H, Sohani SM, Ebrahimian MR, Ebrahimi I, et al. Validation of a Persian-version of Knee injury and Osteoarthritis Outcome Score (KOOS) in Iranians with knee injuries. Osteoarthritis and Cartilage. 2008; 16(10):117882. [DOI:10.1016/j.joca.2008.03.004] [PMID]

[21] Richer N, Saunders D, Polskaia N, Lajoie Y. The effects of attentional focus and cognitive tasks on postural sway may be the result of automaticity. Gait \& Posture. 2017; 54:45-9. [DOI:10.1016/j.gaitpost.2017.02.022] [PMID]

[22] Lee SM, Lee JH. The immediate effects of ankle balance taping with kinesiology tape on ankle active range of motion and performance in the Balance Error Scoring System. Physical Therapy in Sport. 2017; 25:99-105. [DOI:10.1016/j. ptsp.2016.08.013] [PMID]

[23] Broglio SP, Zhu W, Sopiarz K, Park Y. Generalizability theory analysis of balance error scoring system reliability in healthy 
young adults. Journal of Athletic Training. 2009; 44(5):497502. [DOI:10.4085/1062-6050-44.5.497] [PMID] [PMCID]

[24] Mokhtari Nia HR, Kahrizi S, Sanjari MA, Parnian Pour M. [Test-retest reliability of dynamic postural stability measures in healthy and chronic non-specific low back pain groups (Persian)]. Archives of Rehabilitation. 2013; 13(4):8-19.

[25] Salavati M, Hadian MR, Mazaheri M, Negahban H, Ebrahimi I, Talebian S, et al. Test-retest reliabty of center of pressure measures of postural stability during quiet standing in a group with musculoskeletal disorders consisting of low back pain, anterior cruciate ligament injury and functional ankle instability. Gait \& Posture. 2009; 29(3):460-4. [DOI:10.1016/j.gaitpost.2008.11.016] [PMID]

[26] Azadi F, Parnianpour M, Shakeri H, Kazemnejad A, Akbari Kamrani AA, Arab AM, et al. [How many changes in sit to stand-5 repetition test is real in community dwelling older adult and healthy young people (Persian)]. Iranian Journal of Ageing. 2015; 9(4):252-8.

[27] Azadi F, Parnianpour M, Shakeri H, Kazemnejad A, Akbari Kamrani AA, Arab AM, et al. [Relative and absolute reliability of timed up and go test in community dwelling older adult and healthy young people (Persian)]. Iranian Journal of Ageing. 2014; 8(4):56-66.

[28] Wulf G, McNevin N, Shea C. The automaticity of complex motor skill learning as a function of attentional focus. The Quarterly Journal of Experimental Psychology. 2001; 54(4):1143-54. [DOI:10.1080/713756012] [PMID]

[29] Dault MC, Yardley L, Frank JS. Does articulation contribute to modifications of postural control during dual-task paradigms? Cognitive Brain Research. 2003;16(3):434-40. [DOI:10.1016/ S0926-6410(03)00058-2] 
